\title{
The Feasibility Analysis of Low-temperature Waste Heat Thermoelectric Power Generation, Base on Forced Oil Circulation Cooling Transformer
}

\author{
Jiayi Guo \\ Department of Electrical Engineering \\ North China Electric Power University \\ Baoding, Hebei Province,China \\ Guojiayi1203@qq.com
}

\begin{abstract}
This paper is intended to propose a way of utilizing low-temperature waste heat what base on forced oil circulation cooling transformer. Cooling of large power transformers affect the normal operation of the power system. Existing techniques simply cool the transformer, but there is not take full advantage of the low-temperature waste heat generated during cooling. This approach resulted in a lot of energy waste. In this paper we present an approach based on the most commonly used power transformer cooling mode forced oil circulation cooling to take advantage of low-temperature waste heat. In this paper, the feasibility of this approach are discussed mainly from the transformer temperature, plant transformation, temperature conditions, economy,etc. You can say that this method provides a new way of thinking and methods for energy recycling power system. This technology will help improve the efficiency of the transformer, to achieve the purpose of energy saving. It has a strong promotional value in improving aspects of large power transformers.
\end{abstract}

Keywords- power transformer; forced oil circulation; thermoelectric power generation; low-temperature waste heat; energy conservation

\section{INTRODUCTION}

Oil-filled transformers are the most common power transformer in China. With the improvement of electricity demand In the national production and life, transformer voltage level and capacity has been increasing. Because of this reason causing the transformer load is growing, leading to localized overheating transformer vinternal heat transformers and other centralized heat problem. According to a circular manner oil-immersed transformer can be divided into three kinds, namely, natural convection circulation, forced oil circulation and forced oil circulation guide. In these three methods, the mode that forced oil convection circulating pump, whose power is greater than the natural oil circulation, and oil cooling effect is better than the natural circulation. Forced oil circulation guide, which forces oil circuitous guide in the winding,so that the cooling efficiency can be further improved. Large power transformers currently widespread use of forced oil circulation guide approach to cooling in China[1]. Lowtemperature waste heat of power transformer comes from the power grid,which is an important part of the power grid in the energy loss at this stage. So far, this part of the thermal energy resources have not been untapped. If we can find a way to take advantage of low-temperature waste heat transformer cooling oil, which would greatly reduce the energy consumption of power transformers,and improve the efficiency of the transformer.

Thermoelectric power generation technology, as a new generation technology, because of its low power requirements of the temperature, which can use lowtemperature waste heat and has been widely utilized in many areas of production and life. Considering the forced oil circulation is aimed as much as possible to reduce the temperature of transformer oil, and thermoelectric power generation technology can take advantage of this part of low-temperature waste heat. The combination of the two techniques can improve the cooling effect of transformer oil.At the same time it collects the low-temperature waste heat from the transformer oil, and thus play a fully staffed effect. This paper discusses the feasibility of utilization of these two technologies in power transformersand proves its scientific and can be realized.

\section{ANALYSIS OF TRANSFORMER TEMPERATURE RISE}

Temperature rise specifically refers to that the temperature difference between the part that we analyze from external cooling medium. In terms of oil-immersed power transformers is the difference between the temperature measuring portion of the cooling air temperature. Under normal circumstances, in order to ensure the normal operation of the transformer, the transformer's temperature rise limit is: coil temperature $65 \mathrm{~K}$,and oil temperature $55 \mathrm{~K}$. Typically refers to the average annual temperature of 20 degrees Celsius, and the height is no more than 1000 meters above sea level. the transformer's temperature rise limit is the maximum temperature difference when oil-immersed transformers on work. Typically, the temperature of the transformer is not greater than this value, but it will not much smaller than it[2].

In order to better illustrate the load and the temperature rise of large power transformers under normal operating conditions, we have access to the relevant data to 
investigate the $220 \mathrm{kV}$ substation in Shanghai Gubei representative transformer 2010 annual operating data (Figure 1 shown).

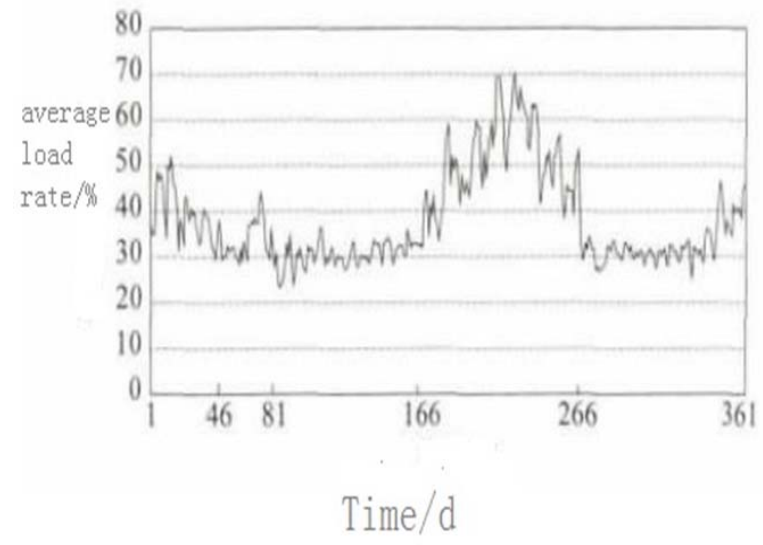

Figure1. Gubei 220kV transformer substation 2010 operating data

The running time of the substation is divided according to the load factor obtained in three stages: The first stage,

Summer (June 17 to September 21, a total of 97 days), this season is the year's largest electricity load season. During this time the average daily load was $51.6 \%$. The second stage, winter (January 1 to March 12, December 6 to 31, a total of 96 days), the average load rate of $34.47 \%$ during this time. The third stage, spring and autumn (the other time was 172 days in total), the average load was $30.61 \%$, within this time. Meanwhile, according to Figure 1, we can see the daily average of transformer operation: the minimum load was $23.28 \%$, the maximum load was $70.28 \%$; the number of days when the load was less than $30 \%$ load factor is 70 days; the number of days when the load was $30 \%$ to $40 \%$ is 147 days; the number of days when the load was $40 \%$ to $50 \%$ is 51 days; the number of days when the load was $50 \%$ to $60 \%$ is 34 days; the number of days when the load was greater than $60 \%$ is 19 days.

At the same time, through access to relevant information, we can get the load and temperature curves 220KV transformer (Figure 2). Thus, we can roughly estimate the water temperature.

By analyzing Figure 2, we can see that: When the transformer is loaded runtime (240MVA), the top oil temperature is about $45 \mathrm{~K}$; when running load is $160 \mathrm{MVA}$, the top oil temperature of about 29K; when running load is 150 MVA, top oil temperature of about 25K. Above projections, the average load factor $51.6 \%$, the top oil temperature is about $25 \mathrm{~K}$, in the summer; when the average load factor was $33.3 \%$, the top oil temperature of about 19K; average load of 34.47 percent, top oil temperature of about $19 \mathrm{~K}$, in the winter.

According to the formula:

oil temperature $=$ temperature rise + ambient temperature

We can estimate the average oil temperature in winter is about $39^{\circ} \mathrm{C}$, and the cooling water outlet temperature is about $39{ }^{\circ} \mathrm{C}$; average summer oil forecast is $61{ }^{\circ} \mathrm{C}$, the cooling water outlet temperature is about $61^{\circ} \mathrm{C}$. Thus, the average annual water temperature is about $50^{\circ} \mathrm{C}[3]$.

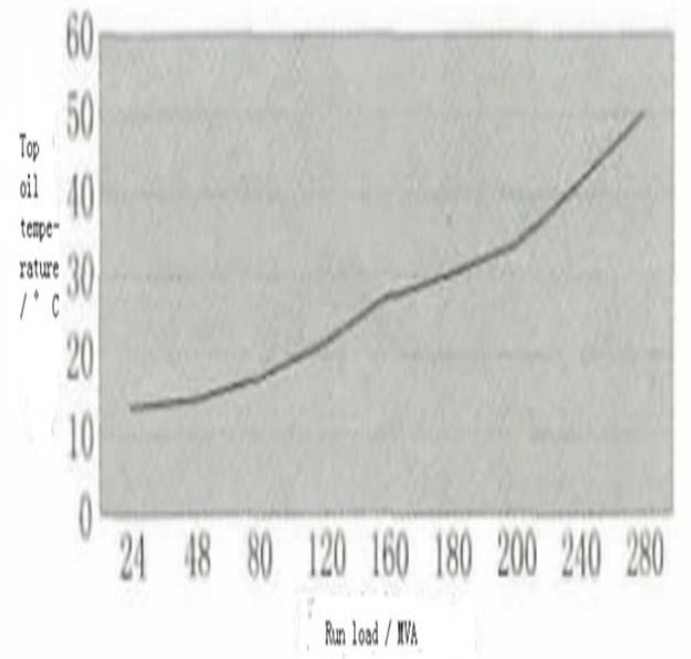

Figure 2. Gubei 220k transformer substation load and top oil temperature curve

\section{THE ALTERATION OF FORCED OIL CIRCULATION PATH.}

At present, China's large transformer cooling mainly uses oil-immersed self-cooled, oil-immersed forced air cooling and air cooling oil circulation, in which air cooling oil circulation is most widely used. Forced oil circulation is a cooling mode,which uses circulating pumps and other components to ransformer oil by pipeline to the cooler,then use water, wind, and other media to reduce the temperature of transformer oil. Finally, the cold transformer oil is transported through the circulation pump back to the internal transformer, thus completing the entire cooling process. Because of the way the forced oil circulation cooling transformer oil export transformer, it is easy to use other media to reduce the temperature of transformer oil. Thus it greatly improves the cooling effect of transformer oil, and it is more conducive to achieve the purpose that reduce the temperature of large power transformers. Therefore this approach is widely used in the current power system. At the same time, this approach is very similar to forced oil circulating water cooling, the differ only in the cooling medium. Large power transformers are now widely used air cooling, instead of water cooling.The main reason is the cost of air-cooled lower than water cooling, but the disadvantage is difficult to collect aircooled cryogenic waste heat. Therefore, we can put this cooling device transformed into forced oil circulation cooling water, so as to achieve the purpose of the collection of low-temperature waste heat. Specific transformation diagram shown in Figure 3 [4]: 


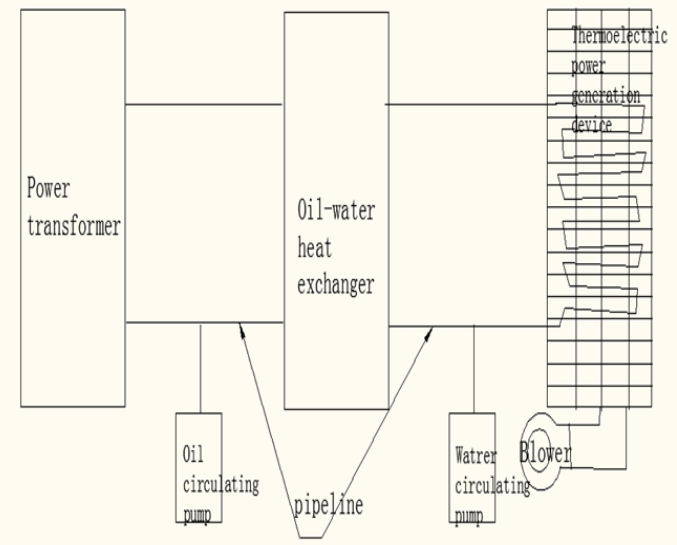

Figure 3. Forced oil circulation path transformation

\section{THE CONDITIONS OF THERMOELECTRIC POWER GENERATION TECHNOLOGY COLLECTING LOW- TEMPERATURE WASTE HEAT}

Semiconductor thermoelectric generation technology mainly used the Seebeck principle, which directly converts heat into electricity. And it gets more and more applications in aerospace, electronics, food reserves and other areas. The ends of two different material conductors connected to the loop in this technique. When the temperature of the binding sites, in the loop there will produce thermal electromotive force (also called thermal emf). [5] Figure 4 is thermocouple experimental schematic diagram, the heavy line $\mathrm{A}$ and thin line $\mathrm{B}$ are different materials of conductor, and the conductor of $\mathrm{A}$ and $\mathrm{B}$ is called the thermocouple. [6] High temperature side $C$ (temperature record for T1) is working side, which usually be tied together; Low temperature side $\mathrm{O}$ (temperature record for T2) is a free end, which would be closed as well. Voltage circuit of temperature difference can be expressed as:

$$
\varepsilon_{0}=b\left(T_{1}-T_{2}\right)
$$

In this formula: $\mathrm{b}$ is the see beck coefficient.

Thermoelectric potential value is basically constant when the work end and free end of thermocouple under the condition of constant temperature, namely thermocouple work at a constant temperature difference[7],

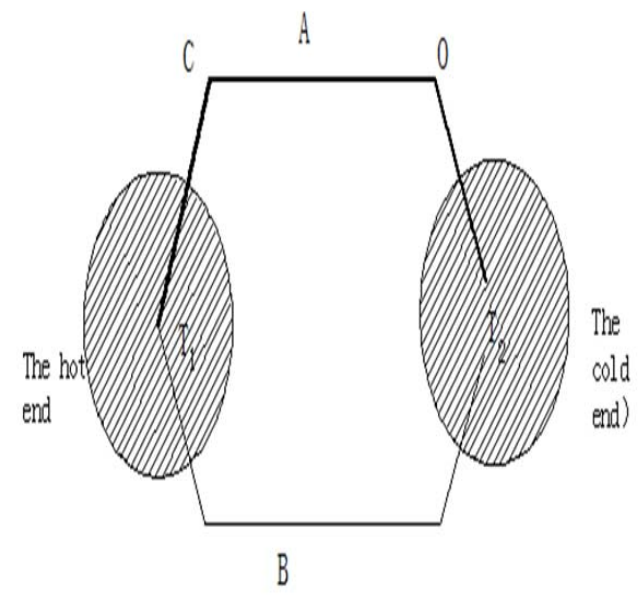

Figure 4. Thermocouple principle diagram
The most suitable temperature difference existing thermocouple power semiconductor thermoelectric power generation piece is about $60 \mathrm{~K}$. It can work at a higher efficiency when the temperature difference is $40-90 \mathrm{~K}$. Device is given in this paper can provide for the generation component of the difference in temperature is about $50 \mathrm{~K}$. Considering the heat diffusion in the transfer process and so on, actually delivered to thermoelectric power generation unit temperature difference of about 45K. The temperature difference conforms to the requirements of Thermoelectric Generator.[8]

\section{THE ECONOMY OF THIS TECHNOLOGY}

Due to the limitations of existing semiconductor materials technology, and now the price is higher thermocouple thermoelectric power generation semiconductor devices.At the same time the power generation efficiency is low.For example the type of SP1848-27145 thermoelectric power generation chip,whose Market retail price is about 15 yuan / piece. Its generating power of about $0.8 \mathrm{~W} /$ tablets when the temperature difference is $45 \mathrm{~K}$. According to the current market price is estimated that about four years to recover costs.It is a long time so that the technology is hardly to spread. However the price of the raw semiconductor material such as silicon ,which is used to make thermocouples Thermoelectric power generation device is not expensive.The reason why it is expensive is poor manufacturing techniques. However, with the development of materials and technology, in the near future, the production cost of semiconductor materials will be greatly reduced. So that this technology can be obtained in large scale.[9]

\section{CONCLUSIONS}

Low-temperature waste heat thermoelectric power generation what base on forced oil circulation cooling transformer. It forced oil circulation cooling pipes and thermoelectric power generation components combine. In the premise of Completing transformer cooling requirements.It put the transformer's low temperature waste hate resources that into electric energy resources. It can reduce the energy loss of the transformer in a certain extent. Thus, it can improve the efficiency of energy transfer transformer, and play the effect of energy conservation. However, this technique is limited to materials technology,so that the cost of this technique is high and it is difficult to promotion of it. [10] However, with the development of materials and technology, in the near future, the production cost of semiconductor materials will be greatly reduced. So that this technology can be obtained in large scale.

\section{REFERENCES}

[1] Xie Rong, Zhang Xing, Guan Liang, Xu Shengli, Forced Oil Circulation Cooling Transformer Internal Temperature Field Numerical Simulation, Electric Power Science and Engineering, Vol. 28 No. pp. 10: 47-52, October 2012.

[2] Liu Zixiang capital, Ooil-immersed Power Transformers Rise of], Guangxi Electric Co., the total on 93: pp. 151-152, December 2007.

[3] Wang Zheng,Huang Lei,Wang Bin,Li Binai, Re-use of waste heat cooled transformer, Power and Energy, Vol. 35, No. 3:pp. 401-404, June 2014. 
[4] Li Xun, Wang Xieyan, 500KV Jing'an Low Water Circulation Cooling System Failure Substation, Power \& Energy, Vol. 33 No. 2: pp. 140-143, April 2012.

[5] Jing-jing Yang and Yong-sheng Liu,et,al,in: Design and Efficiency of Photovoltaic-Thermoelectric Power Generator with Negative Feedback Characteristic, edtied by Journal of Shanghai Jiaotong University, ,47-4(2013),pp. 550-559

[6] $\mathrm{Li} \mathrm{Zu}$ and Qiong Wu,in:Thermoelectric Generation Technology and Its Application in Using Solar Energy. edtied by Nonferrous Metals Engineering \& Research, 31-2(2010),pp. 19-21
[7] Jiayi Guo,Solar Temperature Difference of a Complementary Power Generation Device for Automotive Applications,Applied Energy and Power Engineering IV Part 1:pp. 30-34.

[8] De Chen, Analysis of Cooling System Transformation of $220 \mathrm{kV}$ Forced-oil Type Transformer, Science \& Technology Information, vol. 35,2012,pp. 143-149.

[9] Liu Honglei,Li Guanghua,Wang Dejian,Zhang Bin, Experimental study on thermal model of oil-immersed transformer heat pipe, Huadian Technology, Vol. 35 No. 2,Feb. 2013,pp. 36-38

[10] Yang Lei , Forced air cooling transformer oil circulation system, Innovation Forum \& Innovative Technology,2015,pp. 79. 